This item was submitted to Loughborough's Research Repository by the author.

Items in Figshare are protected by copyright, with all rights reserved, unless otherwise indicated.

\title{
Uncertainty in pore size distribution derived from adsorption isotherms: I. classical methods
}

PLEASE CITE THE PUBLISHED VERSION

http://dx.doi.org/10.1016/j.micromeso.2015.04.026

PUBLISHER

(C) Elsevier

VERSION

AM (Accepted Manuscript)

\section{PUBLISHER STATEMENT}

This work is made available according to the conditions of the Creative Commons Attribution-NonCommercialNoDerivatives 4.0 International (CC BY-NC-ND 4.0) licence. Full details of this licence are available at: https://creativecommons.org/licenses/by-nc-nd/4.0/

\section{LICENCE}

CC BY-NC-ND 4.0

\section{REPOSITORY RECORD}

Madani, S. Hadi, A. Badalyan, Mark J. Biggs, and Phillip Pendleton. 2019. "Uncertainty in Pore Size Distribution Derived from Adsorption Isotherms: I. Classical Methods". figshare.

https://hdl.handle.net/2134/18220. 


\section{Uncertainty in pore size distribution derived from adsorption}

\section{isotherms: I. Classical methods}

S. Hadi Madani ${ }^{a}$, Alexander Badalyan $^{b}$, Mark J. Biggs $^{c, *}$, Phillip Pendleton $^{a, d, \dagger}$

${ }^{a}$ Ian Wark Research Institute, University of South Australia, Mawson Lakes, SA 5095, Australia

${ }^{\mathrm{b}}$ Australian School of Petroleum, University of Adelaide, Adelaide, SA 5005, Australia

c School of Chemical Engineering, University of Adelaide, Adelaide, SA 5005, Australia

${ }^{\mathrm{d}}$ Sansom Institute, University of South Australia, Adelaide, SA 5001, Australia

\footnotetext{
* Current address: School of Science, Loughborough University, Leicestershire, UK LE11 3TU, UK

${ }^{\dagger}$ Corresponding Author: E-mail: Phillip.Pendleton@unisa.edu.au; Tel: +61 (0)8 83022188
} 


\begin{abstract}
Procedures for propagation of uncertainty in pore size distribution calculation based on classical methods for both micro and mesoporous materials are described. Uncertainty in experimental adsorption isotherm data and uncertainty in temperature are introduced as the main sources for uncertainty in height and position of peaks of PSD determined via classical mesopore size distribution determination method. Uncertainty in PSD derived from classical micropore size distribution methods mainly arises from uncertainty in experimental isotherm data. Calculation step size is shown to have some effects on magnitude of uncertainty in micropore calculation. Micropore size distribution calculations are also highly sensitive to the adsorptive molecular diameter.
\end{abstract}

Keywords: adsorption, pore size distribution, uncertainty, porous solids

\title{
1. INTRODUCTION
}

Gas adsorption isotherm measurement is one of a suite of methods applied for surface and internal structure characterization of powders and porous materials. Details derived from these measurements include specific surface area, pore size distribution, pore connectivity, surface chemistry, and the area-density of any surface functional groups [1]. Determination of pore size distribution (PSD) for porous materials is one of the most challenging and important steps in porous materials characterization. Among numerous methods available for PSD determination, those based on adsorption isotherm data are frequently used and reported in the literature. These methods can be classified into two main categories: classical methods and the integral equation approach [1]. Many reviews and publications exist comparing the output of these methods, often showing differences between them in the so-called mean and 
distribution of the pore size. Missing from these and the published literature in general, is an analysis of the contribution to these results of the inherent uncertainty in the physical constants and the experimental data. This paper proposes to fill this gap.

In what we classify as the classical methods for PSD evaluation from gas adsorption isotherms, researchers appreciated there are differences in the pore filling and fluid-solid interactions mechanisms for mesopores and micropores; PSD theory developed separately. In 1870, Thomson (Lord Kelvin) introduced the "Kelvin equation" which became a basis for the interpretation of the hysteresis loop and higher relative pressure ranges of (nitrogen) gas adsorption isotherms [2]. In 1951, Barrett et al. [3] developed the first practical method for mesopore size distribution evaluation, often referred to as the BJH method, using a cylindrical pore model based on the Kelvin equation. Values of adsorbed layer thickness were calculated as a function of relative pressure by Lippens et al. [4]. It was assumed that for the adsorptive, the thickness of adsorbed layer is only a function of relative pressure and independent of the porous solid surface. Dollimore and Heal [5, 6] introduced a modified version of the BJH method and used Halsey's method to calculate adsorbed layer thickness [7]. They also discussed the inaccuracy of the BJH method for small pore sizes caused by assigning incorrect values to constant coefficients used in the method. Later [6], they corrected the coefficient used by Halsey. Overall, the Kelvin equation and methods based on it employ a macroscopic thermodynamics approach and assume a continuous liquid phase in the pores, with defined surface tension, in equilibrium with the gas phase. These assumptions are correct for large mesopores; as the pore width approaches $2 \mathrm{~nm}$ the surface tension of the adsorbed layer must increase to much higher values than equivalent liquid values to maintain the equilibrium pressure [2]. Although some studies show that the Kelvin equation-based methods fail for pore sizes smaller than $3.5 \mathrm{~nm}$ [8], such analyses are applied frequently for pore sizes within the mesopore range (> $2 \mathrm{~nm})$. 
The classical thermodynamics approach to PSD analysis due to Horvath and Kawazoe (HK) is frequently applied to the micropore range [9]. The original HK model was developed for slit pores with a linear Henry's law isotherm assumption. Saito and Foley proposed HKtype equations for cylindrical pores [10]; Cheng and Yang presented the first modified HK model based on the Langmuir isotherm and also included spherical pore equations in their analyses [11]. Rege and Yang (R-Y) addressed the incorrect estimation of adsorbateadsorbate interactions energy in the original HK model which had caused the model to underpredict the PSD for micropore widths exceeding two adsorbate molecular diameters. Their improvements considered molecular-packing effects in micropores and presented a corrected form of the HK model for all three pore geometries: slit, cylinder, and sphere [12].

Many commercial adsorbents, such as selected activated carbons, contain both micropores and mesopores, not as distinct ranges but with one merging into the other, typically deduced from the combination of an enhanced amount adsorbed at low relative pressures leading into Type IV adsorption isotherm shape. For such materials, a detailed PSD analysis via the classical methods needs to be made across two separate, applicable, relative pressure ranges. Often, there is no distinct overlap between the models, particularly in the "transition" width range $1.5-3.0 \mathrm{~nm}$.

The more recently developed PSD determination methods based on those of Seaton and coworkers and followed by other researchers[13-18], employ an adsorption integral equation approach. Intrinsically, these methods include a combination of molecular simulation methods and statistical mechanics. Molecular dynamics, Monte-Carlo simulation, or density functional theory (DFT) have been used as the base models for simulation of the adsorption isotherm on a solid with pre-defined pore sizes represented by single pore isotherms. Calculation of a sufficient number of single pore isotherms combined with adsorption integral equation inversion results in a PSD definition. For a detailed analysis see [19]. Over the last 
three decades a vast number of papers have been published describing the development, modification, and application of adsorption integral equation methods for PSD determination (For a review see [20] or [21]). An advantage these methods have over the classical approaches is their applicability across both micropore and mesopore ranges. On comparison with the classical PSD methods, they are also considered more reliable since they were developed with fewer assumptions. Their application is not ubiquitous; except for nitrogen, argon, and carbon dioxide which are well studied in the literature; reliable parameters and models for other adsorptives are not yet well defined. Even for these well-studied adsorptives, parameters and models are available only for specific temperatures, and are not readily transferable to other adsorption temperatures. Additionally, calculation of single pore isotherms and adsorption integral inversion are sophisticated methods requiring elaborate computer programs. Considering these shortcomings in the new methods, classical models are and should be considered helpful analytical methods for PSD determination.

Experimental results always include a variation in the actual measured parameter, the experimental uncertainty. Gas adsorption measurements incorporate measurement and control of several parameters and uncertainty in each of these parameters contribute to the combined standard uncertainty (CSU) in the results presented as adsorption isotherms. Any subsequent calculations of parameters, such as the BET specific surface area, specific pore volume, or the PSD propagate these uncertainties, leading to larger-valued CSU.

There is a clear difference between the results that we present as uncertainty and other concepts such as accuracy, precision, repeatability, reproducibility, and error [22]. It is important to understand their differences; pedagogically, their interchangeable use is discouraged. Accepted definitions of each are given in Table 1. Unlike "accuracy" which is qualitative, the term we use in this work as "standard uncertainty" is a quantitative parameter. Terms such as precision, repeatability, and reproducibility are used for repetitive 
measurements of experiments and, since we are using single isotherm results, i.e., no repetition, it would be wrong to talk about these terms. The term "error" is defined as the difference between a measurement and the actual or accepted value. In adsorption science, an actual (single) value cannot be defined, thus, in this context error is considered an idealized concept. Uncertainty in a measurement can be defined even when the exact value is not known.

\section{Insert Table 1 here}

Relatively few studies on adsorption uncertainty have been published in the peer-reviewed literature. Loebenstein and Deitz reported values for uncertainty of adsorption data, suggesting large dead spaces in sampling tubes are the main sources of uncertainty in adsorption data and concluded that uncertainty for low specific surface area (SSA) solids is much larger than high SSA solids [23]. They considered the first points of each isotherm for their analysis and reported uncertainties related to materials with different SSAs. In reviewing their work it is important to appreciate that with today's more sensitive, temperature-controlled, electronic capacitance manometers, the uncertainties are much smaller. Ross and Olivier and later Robens et al. addressed uncertainty of adsorption experiments corresponding to apparatus calibration and the non-ideal behaviour of helium and nitrogen, respectively [24, 25]. Effects of liquid nitrogen level control on adsorption results was first reported by Killip et al. in their patent [26].

Using a custom-built automated, manometric gas adsorption apparatus, Badalyan and Pendleton accurately calibrated the dosing and sample volumes of their equipment and demonstrated the effects of their propagated uncertainty on isotherm results $[27,28]$. They also considered the corresponding uncertainties for physical parameters, volume calibration, dead-volume calculations, liquid nitrogen level-control, calculation of amount of gas adsorbed, and sample mass measurement in their study and calculated the CSU for the 
adsorption isotherm data and subsequent BET analysis. They showed cumulative behaviour of CSU in adsorption data with experiment progress resulting in larger uncertainties in higher relative pressure data in the adsorption branch and still larger uncertainties in low pressure data in the desorption branch. They concluded the primary contributions to CSU are sample mass measurement, dead-volume determination, and liquid nitrogen level-control [28, 29]. Badalyan and Pendleton also propagated uncertainty in adsorption data to the CSU of $\alpha_{S}$ analyses and pore volumes determination [30, 31]. Here they showed adsorbent surface chemistry has important influences on the determination of micropore filling and condensation process resulting in uncertainty in micropore volumes.

No published literature exists describing the propagation of isotherm data uncertainty into the pore size distribution for either classical methods or integral equation approaches. However, recently Caguiat et al. calculated PSD based on nitrogen and $\mathrm{CO}_{2}$ adsorption data using commercial software. They calculated PSD using different DFT models and reported effect of DFT model and pore geometry on calculated PSD [32].

Here, we discuss the results of uncertainty propagation into classical PSD analysis methods for mesopores, due to Dollimore and Heal, and micropores, due to Rege and Yang, using well-defined adsorption isotherm data containing combined standard uncertainty. The results of a parametric sensitivity analysis for each method are also discussed. No effort is made to interpret or understand any differences between the PSD for mesopores and micropores in the transition range other than attribute them to differences between their foundation assumptions and pore geometry. Uncertainty propagation in adsorption integral equation methods is presented and discussed elsewhere [19].

\section{EXPERIMENTAL}


Single sheet, plain-weaved, activated carbon cloth FM1/250 (ex. Calgon Carbon, Pittsburgh, PA, USA) was used as an exemplar slit-shaped micropore material and MCM-41 as a cylinder-shaped mesopore. The MCM-41 sample was prepared by a hydrothermal synthesis method at room temperature [33]. Nitrogen gas adsorption experiments were carried out at $77 \mathrm{~K}$ using an automated manometric gas adsorption apparatus described elsewhere [27]. Prior to measurements, samples were degassed at $200{ }^{\circ} \mathrm{C}$ and a background vacuum of $0.1 \mathrm{mPa}$ for $8 \mathrm{~h}$. Thermal transpiration corrections were applied for pressures below $266 \mathrm{~Pa}$. Throughout the adsorption-desorption process the liquid nitrogen level was controlled constant ( $\pm 0.15 \mathrm{~mm})$. Ultra-high purity (99.999\%) helium and nitrogen from BOC Gases, Australia were used for dead-space and adsorption isotherm measurements, respectively.

\section{Insert Table 2 here}

\section{RESULTS AND DISCUSSION}

To discuss the sources of uncertainty in classical PSD determination methods, we focus on the corrected BJH method proposed by Dollimore and Heal [5, 6] for mesopore size distribution analysis and on the corrected HK method proposed by Rege and Yang (R-Y method) [12] for micropore size distribution analysis. Evaluation of the uncertainty in adsorption isotherm data was clearly demonstrated by Badalyan and Pendleton [28]. This uncertainty is expected to be propagated as a major contribution to the uncertainty in subsequent PSD analyses. Additional sources of uncertainty are expected to be due to the physical constants used in such analyses. Table 2 lists the physical constants used for calculations, their accepted values and, where available, their standard uncertainty.

To calculate CSU $\left(u_{c}\right)$ in a variable from several independent variables, a multi-variable uncertainty propagation method is used [37].The general formula is given as: 


$$
y=f\left(x_{1}, x_{2}, \ldots x_{n}\right) \longrightarrow u_{C}(y)=\sqrt{\left[\left(\frac{\partial y}{\partial x_{1}}\right)_{i=2, \ldots, n} U x_{1}\right]^{2}+\cdots+\left[\left(\frac{\partial y}{\partial x_{n}}\right)_{i=1, \ldots, n-1} U x_{n}\right]^{2}}
$$

Here, $u_{C}(y)$ would be the CSU calculated from the uncertainty in the variables $x_{1}, x_{2}, \ldots x_{n}$, quoted as $U x_{1}, U x_{2}, \ldots U x_{n}$.

The two adsorbents were selected because each exhibits distinct pore size ranges. The FM1/250 shows a sharp, narrow PSD in the micropore range [38] and MCM-41 shows a sharp, narrow PSD in the mesopore range with no influence from any inherent micropores. We suggest a narrow distribution of width is important to demonstrate clearly any influence of factors contributing to subtle changes in either the position of the maximum in the peak in the PSD and/or the height of the peak. The latter relates to the amount or number of pores found within a selected calculation-width.

Figure 1. shows high resolution nitrogen adsorption isotherms on these two adsorbents. Applying the Badalyan and Pendleton method [28], uncertainty in the experimental data was calculated and shown as uncertainty bars in each amount adsorbed. In all cases, the uncertainty in the relative pressure was negligible, and is not included. The cumulative nature of the uncertainty in the adsorption and desorption data is inherent in the method of isotherm data collection; the smallest uncertainty occurs in the first data point, increasing in value with increasing number of data points collected. In the present analyses, the largest uncertainty in the amount adsorbed in the adsorption branch occurred for that value closest to the saturation pressure. Since desorption data are typically obtained after the adsorption process, the desorption branch defined for the MCM-41 analysis shows an increasing uncertainty in the amount adsorbed. From an understanding of the classical methods of PSD analysis, it follows that the uncertainty corresponding to experimental data in micropore analysis would be 
expected to increase with increasing pore size; assuming the desorption branch is used for mesopores, the uncertainty corresponding to experimental data in mesopore analysis would be expected to increase with decreasing pore size.

\subsection{Mesopore analysis}

The mesopore cumulative volume and size distribution shown in Figure 2 were calculated via the Dollimore and Heal method including the corrected Halsey coefficient for adsorbed layer thickness $[5,6]$. The vertical uncertainty bar intensities for both analyses are relatively larger in value for the smallest pore sizes, decreasing with increasing pore size. A sensitivity analysis of the calculation procedures indicated the principal source of uncertainty in these analyses was in the experimental isotherm data, contributing $99.7 \%$ to the total CSU. The desorption branch of the adsorption isotherm is most often used for PSD evaluation; if the adsorption branch were selected, the intensity of the vertical uncertainty values would be reversed. In contrast, the uncertainty in the pore width (at each value) increases with increasing width. The Dollimore and Heal method calculates a pore radius $(r)$ as the summation of the Kelvin radius $\left(r_{K}\right)$ and the adsorbed layer thickness on the pore wall $(t)$, as Eq. (2):

$$
r=r_{k}+t
$$

Each of the independent variables in Eq. (2) depends on relative pressure and/or temperature, thus, pore size is independent of uncertainty in the amount adsorbed. Since the adsorbed layer thickness is a function of relative pressure and the uncertainty in relative pressure is small (precision of the MKS pressure transducers used as part of the adsorption apparatus was $0.05 \%$ of scale reading), uncertainty corresponding to the physically adsorbed layer is relatively small compared to the $u_{C}(r)$. The Kelvin radius is related to adsorbate 
surface tension $(\gamma)$, adsorbate molar volume $\left(V_{m}\right)$, universal gas constant $(R)$, (sample) temperature $(T)$, and relative pressure $\left(p / p^{o}\right)$ via the Kelvin equation:

$\ln \left(p / p^{o}\right)=\frac{-2 \gamma V}{r_{K} R T} \cdot \cos \theta$

The condensed, liquid-like, adsorbed (multiple) layer is always regarded as being in contact with, at least, the first statistical adsorbed layer and thus, the contact angle $(\theta)$ is evaluated as zero. From the constants in Table 2, the relative uncertainty in temperature is largest. Adsorbate surface tension and molar volume, which is typically derived from the fluid density, are also temperature dependent; these two variables also have large relative uncertainty.

Based on Eq. (1), the CSU in the Kelvin radius would be evaluated via:

$u_{C}\left(r_{K}\right)=\sqrt{\left[\frac{\partial\left(r_{K}\right)}{\partial(\gamma)} U(\gamma)\right]^{2}+\left[\frac{\partial\left(r_{K}\right)}{\partial(V)} U(V)\right]^{2}+\left[\frac{\partial\left(r_{K}\right)}{\partial(T)} U(T)\right]^{2}+\left[\frac{\partial\left(r_{K}\right)}{\partial(R)} U(R)\right]^{2}+\left[\frac{\partial\left(r_{K}\right)}{\partial\left(p / p^{o}\right)} U\left(p / p^{o}\right)\right]^{2}}$

The contributions from relative pressure and universal gas constant are relatively small and thus, after calculation and substitution of derivatives, uncertainty in Kelvin radius can be rewritten as:

$u_{C}\left(r_{K}\right)=\frac{-2}{\ln \left(p / p^{o}\right) R T} \sqrt{[-V U(\gamma)]^{2}+[\gamma U(V)]^{2}+\left[\frac{\gamma V}{T} U(T)\right]^{2}}$

Clearly, temperature control is important in the evaluation of the uncertainty in the Kelvin radius. What causes the uncertainty to increase with increasing radius value? The sample temperature is relatively constant for each of the experimental points; the square root term in Eq. (5) is relatively constant. While the relative pressure increases, $\ln \left(p / p^{o}\right)$ decreases in (negative) magnitude and, since this term is a denominator, the CSU in pore radius increases. This result is clear in Figure 2. 
In conclusion, our calculations show for a classical mesopore size distribution, uncertainty in experimental adsorption isotherm data is the principal source of uncertainty in the intensity of the PSD and in the cumulative volume. Uncertainty in pore size (or width) values is influenced by uncertainty in sample temperature. One way to reduce uncertainty in the intensity of the PSD peaks would be to decrease uncertainty in the experimental data, achieved by intermittent adsorption isotherm measurement. By dividing the adsorption isotherm into several relative pressure-defined segments, with each measured independently, i.e. the sample would be re-evacuated after completion of the adsorption analysis for each segment, the subsequent cumulative uncertainty in the amount adsorbed along the isotherm would be reduced. The calculated PSD from such experimental data would then exhibit smaller-valued uncertainty in peak intensity. To reduce uncertainty in peak position within the PSD, uncertainty in sample temperature needs to be reduced. Sample temperature is calculated from atmospheric pressure; uncertainty in sample temperature is a function of the uncertainty in the barometer used for pressure determination. The experiment as a whole is susceptible to fluctuations in atmospheric pressure. To reduce uncertainty in sample temperature higher precision barometers need to be used for atmospheric pressure measurement. Ideally, the sample and the liquid nitrogen bath would be surrounded by an isobaric chamber.

\subsection{Micropore analysis}

Figure 3 shows the calculated cumulative volume and PSD for the microporous FM1/250 using the R-Y method [12]. The CSU in cumulative volume, PSD, and pore size are included. The calculation details and uncertainty propagation procedures are summarized in the Supplementary Information. Each plot includes the dependent variable CSU. The relatively small CSU for the cumulative volume adsorbed by the smallest pores clearly increases with 
increasing pore width. This increase is also due to cumulative contributions. The principal contribution to these derived from the CSU in the experimental amount adsorbed data. The CSU in the (intensity) data as the micropore size distribution also increases with increasing pore width.

Micropores are typically of width $<2.0 \mathrm{~nm}$. Using the R-Y methodology, this width is equivalent to a pressure $\approx 0.16 p^{\circ}$. For the current sample, the maximum in the distribution occurs at $\approx 0.6 \mathrm{~nm}$, equivalent to a pressure of $4 \times 10^{-4} p^{\circ}$. Reproducible adsorption isotherm data collection at these low pressures is challenging, with most published isotherms comprising $<30$ adsorption points below $0.16 p^{\circ}$. The $u_{C}\left(V_{a d s}\right)$ at these low pressures is usually relatively small, with their contributions to the PSD reflected as relatively small values increasing in value across the PSD with increasing pore width. As with the mesopore analyses, temperature control has the greatest effect on the physical properties used in the RY analysis. Nonetheless, sensitivity analysis including these coefficients amount to only $1.2 \%$ of the CSU with the remaining $98.8 \%$ due to CSU in the experimental data.

The CSU in the pore width $\left(u_{C}(\right.$ width $)$ ) associated with each data point is also included in the PSD plot in Figure 3. Pore size is principally a function of the relative pressure, with the remaining coefficients making negligible contributions to $u_{C}$ (width). Their values are not visible in the main plot; the inset in Figure 3 shows $u_{C}$ (width) for the data point equivalent to $0.57 \mathrm{~nm}$, where the relative uncertainty in width is $0.0068 \%$ and the relative uncertainty in intensity is $1.05 \%$.

In conclusion, since the physical constants are well defined with relatively small uncertainties and the CSU in amount adsorbed and relative pressure in the low pressure range used for the R-Y method are also relatively small, the CSU in the data defining the PSD are relatively small for the narrowest pores but increase with increasing pore width. 
The PSD is the first derivative of the cumulative volume adsorbed $(d V)$ with respect to the pore width ( $d w)$, as $d V / d w$. Intuitively, one would expect uncertainty in both parameters to influence the CSU in this derivative via Eq. (4):

$$
u_{C}(P S D)=\sqrt{\left[\left(\frac{\partial\left(\frac{d V}{d w}\right)}{\partial(d V)}\right)_{d w} U(d V)\right]^{2}+\left[\left(\frac{\partial\left(\frac{d V}{d w}\right)}{\partial(d w)}\right)_{d V} U(d w)\right]^{2}}
$$

Since uncertainty in pore size is small, the second term is $<<$ first term, thus,

$$
u_{C}(P S D)=\frac{U(d V)}{d w}
$$

The ranges applicable to the gradient analysis are defined by the curves representing the upper and lower limits of the CSU in the cumulative volume adsorbed and the width of the “step size” (as $d w$ ). Eq. 5 shows dependency of uncertainty in PSD to uncertainty in cumulative volume $(V)$ and calculation step size $(d w)$. As expected from Eq. 5, our analysis identified selecting smaller step sizes in the PSD calculation would result in larger CSU in the calculated PSD. However when the CSU in the amount adsorbed was small, the CSU in the cumulative amount adsorbed was (relatively) small and, regardless of the step size, which must be bounded by the limits of the CSU cumulative amounts adsorbed curves, $u_{C}(P S D)$ was also relatively small.

Like mesopore analysis, one method to reduce uncertainty in calculated micropore size distribution is to decrease uncertainty in the experimental data, achieved by intermittent adsorption isotherm measurement. The calculated PSD from such experimental data would then exhibit smaller-valued uncertainty.

\subsection{Probe molecule dimension effect on micropore size distribution}


Of all the parameters tested above, the accepted physical diameter of nitrogen was held constant. A sensitivity analysis of this variable is quite enlightening in its influence on the PSD. It is well understood that the uncertainty in the accepted value for molecular nitrogen may be due to its method of determination or, as in the case of the constraining environment of a micropore, due to physical compressibility. The accepted molecular diameter of nitrogen is $0.3 \mathrm{~nm}$, a value often cited from Horvath and Kawazoe’s paper[9], which was sourced from an earlier publication. This value is usually referred to as the van der Waals diameter[39-41]. Other van der Waals diameters are available for nitrogen, ranging from $0.292 \mathrm{~nm}$ [42] up to $0.37 \mathrm{~nm}[43]$. A review on reported values is due to Batsanov[43].

Although the relative uncertainty of the coefficients and measurement variables for the R-Y methodology is $<<1 \%$, for a sensitivity analysis applicable to molecule dimensions, we suggest a $1 \%$ variation would be acceptable, giving diameters in the range $0.297-0.303 \mathrm{~nm}$, statistically consistent with those reported[43]. Figure 4a shows the effect of this range on the cumulative amount adsorbed and the PSD for each of the three diameters. For clarity, the uncertainty bars are excluded from the whole pore range, but are included in the inset showing the variation in the position and intensity of the (primary) peak in each distribution.

Considering only a $1 \%$ change in molecular dimension, and the calculated CSU for cumulative volume was small (Figure 3), Figure 4a suggests that the effect of probe molecule dimensions on PSD determination is important. The largest cumulative volume adsorbed corresponds to the largest probe size, $0.303 \mathrm{~nm}$, and the smallest volume for the smallest probe. This observation is counter-intuitive. A smaller probe would penetrate and accumulate in pores too narrow for a larger probe across the relative pressure range examined, as a molecular sieve process. The reason for this apparent anomaly lies in two combined sources. Firstly, in the algorithm used in the R-Y analysis, and secondly, the amounts adsorbed in the isotherm are a set of fixed values, regardless of the probe dimensions. The algorithm, 
summarized in Supplementary Information, requires matching the pore size with the relative pressure, then identifying the volume adsorbed at this matching relative pressure. Assuming no micropore-induced compression of the probe, the minimum pore width must be equivalent to the probe diameter. With an increasing initial probe dimension, one obtains an increasing initial-valued relative pressure and, since the data set as the volume adsorbed is fixed for a particular molecule dimension $(0.3 \mathrm{~nm})$, the initial volume adsorbed for this relative pressure will also increase, and vice versa. Based on this analysis, the intensity of the peaks increased with increasing probe dimensions, but its position was shifted to smaller pore sizes. To have a feeling of how the peak position is changed with the probe dimension, Figure 4b shows the peak position as a function of probe molecular diameter ranging within the reported values in the literature (from $0.292 \mathrm{~nm}$ to $0.370 \mathrm{~nm}$ ). Interestingly, the Figure suggests a linear dependency of peak position to the probe molecular diameter. The decreasing trend in Figure $4 \mathrm{~b}$ is consistent with the data reported in Figure 4a.

A comparison between Figure 4 and Figure 3 is interesting. Figure 3 shows that uncertainty in the physical parameters and also uncertainty in experimental data does not influence the position and/or intensity of the primary distribution peak. On the other hand Figure 4 shows that even $1 \%$ change in molecular diameter will result in considerable change in both intensity and position of the primary peak. This latter observation has particular ramifications for adsorptives whose dimensions are not yet agreed upon.

We emphasise that the above analysis is not an analysis of molecular sieve effects because these are defined as exclusion of pore penetration due to probe molecular dimensions. The amount adsorbed by a micropore for a given relative pressure will always be fixed by the physical dimension of the probe; the major question then becomes an accepted definition of the (correct) molecular dimensions. Secondly, no account is taken of possible (accepted) kinetic diameter-reduction caused by micropore-induced compressibility, as suggested by 
Everett and Powl [44]. Such issues become more important for larger molecules typically employed as molecular sieve probes, including hydrocarbons and $\mathrm{CCl}_{4}$ and $\mathrm{SF}_{6}$.

\section{CONCLUSIONS}

Uncertainty in experimental adsorption isotherm data and uncertainty in physical parameters are considered to be the main sources of uncertainty in PSD determination. Detailed analysis of mesopore size distribution calculations via the Dollimore and Heal method showed how the uncertainty in adsorption data was the main source of uncertainty in peak height or intensity in the PSD. Uncertainty in peak position mainly arose from uncertainty in temperature and was independent of the experimental data. For an improved accuracy in mesopore size distribution analysis we recommend intermittent adsorption isotherm measurement which would lead to decreased uncertainty in the PSD intensity. From an equipment perspective, one would require improved sensitivity in monitoring atmospheric pressure conditions to determine exact positions of the PSD peaks.

For the micropore size distribution analyses, calculations using R-Y method showed that although uncertainty in experimental isotherm data was the principal source of uncertainty in PSD, it only affected peak height; peak positions were relatively stable. Although the step size (value) selection in the calculations was not a source of the uncertainty, it affected the magnitude of the uncertainty. To determine a more accurate micropore size distribution, we again recommend intermittent adsorption isotherm measurement together with optimal selection of calculation step size. Selection of step size is not without consequence; larger step sizes would result in smaller uncertainty and decreased resolution of the PSD, and vice versa. Our suggestion is to select the largest possible step size which gives a reasonable PSD resolution.

The calculated PSD was very sensitive to the probe molecule diameter. Our calculations showed a relative uncertainty as small as $1 \%$ in the molecular diameter would result in 
different peak positions and intensities within the PSD. If a large molecule with no welldefined size and/or with several chemical bonds and compressibility issues were to be used for micropore size distribution analysis, interpretation of micropore size distributions derived from such adsorptives should be made with careful consideration of molecular size effects.

\section{ACKNOWLEDGMENTS}

The authors thank the Australian Research Council discovery program (DP110101293) for funding support and S.H.M also thanks the University of South Australia for a postgraduate research scholarship.

\section{REFERENCES}

[1] F. Rouquerol, J. Rouquerol, K. Sing, Adsorption by powders and porous solids, Academic Press, London, 1999.

[2] S.J. Gregg, Sing, K. S. W, Adsorption, surface area, and porosity, Academic Press, New York, 1967.

[3] E.P. Barrett, L.G. Joyner, P.P. Halenda, The determination of pore volume and area distributions in porous substances. I. computations from nitrogen isotherms, J. Am. Chem. Soc., 73 (1951) 373-380.

[4] B.C. Lippens, B.G. Linsen, J.H.d. Boer, Studies on pore systems in catalysts I. The adsorption of nitrogen; apparatus and calculation, J. Catal., 3 (1964) 32-37.

[5] D. Dollimore, G.R. Heal, An improved method for the calculation of pore size distribution from adsorption data, J. Appl. Chem., 14 (1964) 109-114. 
[6] D. Dollimore, G.R. Heal, Pore-size distribution in typical adsorbent systems, J. Colloid Interface Sci., 33 (1970) 508-519.

[7] G. Halsey, Physical adsorption on non-uniform surfaces, The J. Chem. Phys., 16 (1948) 931-937.

[8] C.M. Lastoskie, N. Quirke, K.E. Gubbins, Chapter 15. Structure of porous adsorbents: Analysis using density functional theory and molecular simulation, in: W. Rudziński, W.A. Steele, G. Zgrablich, Stud. Surf. Sci. Catal., Elsevier, 1997.

[9] G. Horvath, K. Kawazoe, Method for the calculation of effective pore size distribution in molecular sieve carbon, J. Chem. Eng. Jpn., 16 (1983) 470.

[10] A. Saito, H.C. Foley, Curvature and parametric sensitivity in models for adsorption in micropores, AIChE J., 37 (1991) 429-436.

[11] L.S. Cheng, Y. Ralph T, Improved Horvath—Kawazoe equations including spherical pore models for calculating micropore size distribution, Chem. Eng. Sci., 49 (1994) 25992609.

[12] S.U. Rege, R.T. Yang, Corrected Horváth-Kawazoe equations for pore-size distribution, AIChE J., 46 (2000) 734-750.

[13] N.A. Seaton, J.P.R.B. Walton, N. quirke, A new analysis method for the determination of the pore size distribution of porous carbons from nitrogen adsorption measurements, Carbon, 27 (1989) 853-861.

[14] P. Tarazona, Erratum: Free-energy density functional for hard spheres, Phys. Rev. A, 32 (1985) 3148-3148. 
[15] P. Tarazona, Free-energy density functional for hard spheres, Phys. Rev. A, 31 (1985) 2672-2679.

[16] R. Evans, U.M.B. Marconi, P. Tarazona, Fluids in narrow pores: Adsorption, capillary condensation, and critical points, J. Phys. Chem., 84 (1986) 2376-2399.

[17] R. Evans, U.M.B. Marconi, P. Tarazona, Capillary condensation and adsorption in cylindrical and slit-like pores, J. Chem. Soc., Farad. T. 2, 82 (1986) 1763-1787.

[18] P. Tarazona, U.M.B. Marconi, R. Evans, Phase equilibria of fluid interfaces and confined fluids, Mol. Phys., 60 (1987) 573-595.

[19] S.H. Madani, L.F. Herrera, M.J. Biggs, P. Pendleton, Uncertainty in pore size distribution derived from adsorption isotherms: II. Adsorption integral approach, submitted for Micropor. Mesopor. Mat., (2014).

[20] D.D. Do, H. D. Do, Pore characterization of carbonaceous materials by DFT and GCMC Simulations: A review, Adsorpt. Sci. Technol., 21 (2003) 389-423.

[21] J. Landers, G.Y. Gor, A.V. Neimark, Density functional theory methods for characterization of porous materials, Colloid. Surface A., 437 (2013) 3-32.

[22] B.N. Taylor, C.E. Kuyatt, Guidelines for evaluating and expressing the uncertainty of NIST measurement results, in: NIST Technical Note 1297, National Institute of Standards and Technology, 1994.

[23] W.V. Loebenstein, V.R. Deitz, The magnitude of the experimental error in the adsorption of nitrogen at 78K on various surfaces, The J. Chem. Phys., 15 (1947) 687-688.

[24] S. Ross, J.P. Olivier, On physical adsorption, Interscience Publishers, Sydney, 1964. 
[25] E. Robens, J.U. Keller, C.H. Massen, R. Staudt, Sources of error in sorption and density measurements, J. Therm. Anal. Calorim., 55 (1999) 383-387.

[26] G.R. Killip, R.W. Camp, C. Orr, Temperature controlling apparatus for use with pore volume and surface area analyzers Re-issued U.S. Patent 33,567, April 9, 1991 (original U.S. Patent 4,693,124, September 15 1987)

[27] A. Badalyan, P. Pendleton, H. Wu, Development of an automated gas adsorption apparatus for the characterization of the surface area, pore size distribution, and density of powdered materials, Rev. Sci. Instrum., 72 (2001) 3038-3045.

[28] A. Badalyan, P. Pendleton, Analysis of uncertainties in manometric gas-adsorption measurements. I: Propagation of uncertainties in BET analyses, Langmuir, 19 (2003) 79197928.

[29] P. Pendleton, A. Badalyan, Gas adsorption data uncertainty and propagation analyses, Adsorption, 11 (2005) 61-66.

[30] A. Badalyan, P. Pendleton, Uncertainty in $\alpha_{\mathrm{s}}$ analyses and pore volumes propagated from uncertainty in gas adsorption data, in: P.L. Llewellyn, F. Rodriquez-Reinoso, J. Rouqerol, N. Seaton Stud. Surf. Sci. Catal., Elsevier, 2007, pp. 383-388.

[31] A. Badalyan, P. Pendleton, Analysis of uncertainties in manometric gas-adsorption measurements: II. Uncertainty in -analyses and pore volumes, J. Colloid Interface Sci., 326 (2008) 1-7.

[32] J.N. Caguiat, D.W. Kirk, C.Q. Jia, Uncertainties in characterization of nanoporous carbons using density functional theory-based gas physisorption, Carbon, 72 (2014) 47-56. 
[33] S-Y. Park, M. Barton, P. Pendleton, Mesoporous silica as a natural antimicrobial carrier, Colloid. Surface. A, 385 (2011) 256-261.

[34] P.J. Mohr, B.N. Taylor, D.B. Newell, CODATA recommended values of the fundamental physical constants: 2010, in, National Institute of Standards and Technology, 2012.

[35] M.E. Wieser, T.B. Coplen, Atomic weights of the elements 2009 (IUPAC Technical Report), Pure Appl. Chem., 83 (2011).

[36] R.H. Perry, D.W. Green, Perry's Chemical Engineer's Handbook, McGraw-Hill, New York, 1999.

[37] J.R. Taylor, An introduction to error analysis: the study of uncertainties in physical measurements, University Science Books, Sausalito, 1982.

[38] A. Badalyan, R. Bromball, P. Pendleton, W. Skinner, An assessment of activated carbon cloth microporosity change due to chemical activation, Carbon, 48 (2010) 1004-1011.

[39] L. Pauling, The nature of the chemical bond, Cornell University, Ithaca, 1939.

[40] J.D. Dunitz, A. Gavezzotti, Attractions and repulsions in molecular crystals: What can be learned from the crystal structures of condensed ring aromatic hydrocarbons?, Acc. Chem. Res., 32 (1999) 677-684.

[41] G. Filippini, A. Gavezzotti, Empirical intermolecular potentials for organic crystals: the `6-exp' approximation revisited, Acta Crystallogr. B, 49 (1993) 868-880.

[42] A. Bondi, van der Waals volumes and radii, J. Phys. Chem., 68 (1964) 441-451.

[43] S.S. Batsanov, van der Waals radii of elements, Inorg. Mater., 37 (2001) 871-885. 
[44] D.H. Everett, J.C. Powl, Adsorption in slit-like and cylindrical micropores in the henry's law region. A model for the microporosity of carbons, J. Chem. Soc., Faraday Trans. 1, 72 (1976) 619-636. 


\section{TABLES}

Table 1. Definitions used for interpretation of experimental data [22]

\begin{tabular}{|l|l|l|}
\hline Term & Definition & Nature \\
\hline Accuracy & $\begin{array}{l}\text { Closeness of the agreement between the result of } \\
\text { a measurement and the value of the measurand } \\
\text { (True value) }\end{array}$ & Qualitative \\
\hline Precision & $\begin{array}{l}\text { The closeness of agreement between independent } \\
\text { test results obtained under stipulated conditions. } \\
\text { Precision encompass both repeatability and } \\
\text { reproducibility as they may be called precision in } \\
\text { repeatability and precision in reproducibility. }\end{array}$ & Quantitative/Qualitative \\
\hline Repeatability & $\begin{array}{l}\text { Closeness of the agreement between the results } \\
\text { of successive measurements of the same } \\
\text { measurand carried out under the same conditions } \\
\text { of measurement (e.g. adsorption measurement on } \\
\text { the same material with the same equipment in the } \\
\text { same operating conditions including location, } \\
\text { temperature, and pressure). }\end{array}$ & \\
\hline Reproducibility & $\begin{array}{l}\text { Closeness of the agreement between the results } \\
\text { of measurements of the same measurand carried } \\
\text { out under changed conditions of measurement } \\
\text { (e.g. adsorption measurements on the same } \\
\text { material carried out using different instrument or } \\
\text { with different methods or in different locations, } \\
\text { temperatures or pressures). }\end{array}$ & \\
\hline Error & $\begin{array}{l}\text { Result of a measurement minus the actual value } \\
\text { (or accepted conventional value) of the } \\
\text { measurand. }\end{array}$ & Quantitative \\
\hline
\end{tabular}


Table 2. Physical constants and their uncertainty used for classical PSD determinations

\begin{tabular}{|c|c|c|c|c|c|}
\hline Symbol & Parameter & Value & $\begin{array}{l}\text { Standard } \\
\text { uncertainty }\end{array}$ & Unit (SI) & Data source* \\
\hline $\mathrm{T}$ & Sample temperature & 77.497 & 0.010 & K & [28] \\
\hline $\mathrm{R}$ & Universal gas constant & 8.3144621 & $7.5 \times 10^{-6}$ & $\mathrm{~J} \cdot \mathrm{mol}^{-1} \cdot \mathrm{K}^{-1}$ & [34] \\
\hline $\mathrm{M}_{\mathrm{N}_{2}}$ & $\begin{array}{l}\text { Molecular weight, } \\
\text { nitrogen }\end{array}$ & $28.01371 \times 10^{3}$ & $8.5 \times 10^{-4}$ & kg.mol ${ }^{-1}$ & [35] \\
\hline $\mathrm{m}_{\mathrm{e}}$ & Electron mass & $9.10938291 \times 10^{-31}$ & $4 \times 10^{-38}$ & $\mathrm{~kg}$ & [34] \\
\hline c & Light speed & 299792458 & 0 & $\mathrm{~m} \cdot \mathrm{s}^{-1}$ & [34]/Definition \\
\hline$\alpha_{a}$ & $\begin{array}{c}\text { Polarizability of } \\
\text { adsorbent (carbon) }\end{array}$ & $1.46 \times 10^{-30}$ & $\mathrm{n} / \mathrm{a}^{* *}$ & $\mathrm{~m}^{3}$ & [9] /- \\
\hline$\alpha_{\mathrm{s}}$ & $\begin{array}{c}\text { Polarizability of } \\
\text { adsorptive (nitrogen) }\end{array}$ & $1.02 \times 10^{-30}$ & $\mathrm{n} / \mathrm{a}^{* *}$ & $\mathrm{~m}^{3}$ & [9] /- \\
\hline$\chi_{a}$ & $\begin{array}{l}\text { Magnetic susceptibility } \\
\text { (adsorbent atom, carbon) }\end{array}$ & $2 \times 10^{-35}$ & $\mathrm{n} / \mathrm{a}^{* *}$ & $\mathrm{~m}^{3}$ & [9] /- \\
\hline$\chi_{\mathrm{s}}$ & $\begin{array}{l}\text { Magnetic susceptibility } \\
\text { (adsorptive, nitrogen) }\end{array}$ & $13.5 \times 10^{-35}$ & $\mathrm{n} / \mathrm{a}^{* *}$ & $\mathrm{~m}^{3}$ & [9] /- \\
\hline$\rho_{\mathrm{N}_{2}}$ & Density, liquid nitrogen & 807.2395 & 0.0464 & $\mathrm{~kg} \cdot \mathrm{m}^{-3}$ & $\begin{array}{c}\text { [36] /Calculated based } \\
\text { on uncertainty in } \mathrm{T}\end{array}$ \\
\hline $\mathrm{N}_{\mathrm{A}}$ & Avogadro number & $6.02214129 \times 10^{23}$ & $2.7 \times 10^{16}$ & $\mathrm{~mol}^{-1}$ & [34] \\
\hline$\gamma$ & $\begin{array}{c}\text { Surface tension, liquid } \\
\text { nitrogen }\end{array}$ & $8.837 \times 10^{-3}$ & $3 \times 10^{-6}$ & $N \cdot m^{-1}$ & $\begin{array}{c}\text { [36] / Calculated based } \\
\text { on uncertainty in } \mathrm{T}\end{array}$ \\
\hline
\end{tabular}

* The information given is cited as the reference for the value / the reference for the uncertainty

** No values could be found in the literature; uncertainty for this parameter is considered negligible. 


\section{FIGURE CAPTIONS}

Figure 1. Nitrogen adsorption isotherm for microporous carbon cloth FM1/250 (-) and mesoporous MCM-41 (adsorption: - -, desorption: •••) showing uncertainty in amounts adsorbed for each equilibrium relative pressure. Each sample was degassed at $200{ }^{\circ} \mathrm{C}$ and 0.1 $\mathrm{mPa}$ for $8 \mathrm{~h}$

Figure 2. Calculated cumulative (liquid) volume adsorbed $(--)$, PSD (-), and related uncertainties for mesoporous MCM-41

Figure 3. Calculated cumulative (liquid) volume adsorbed (- - ), PSD (-), and related uncertainties for microporous carbon cloth FM1/250

Figure 4. Calculated cumulative volume (- - ) and PSD (-) for microporous carbon cloth using different nitrogen molecular diameters: $0.297(\bullet), 0.3(\mathrm{x})$, and $0.303(\mathbf{\square})$. The inset shows the subtle increase in (apparent) mean pore diameter for the smallest probe dimension, $0.297 \mathrm{~nm}$. 


\section{FIGURES}

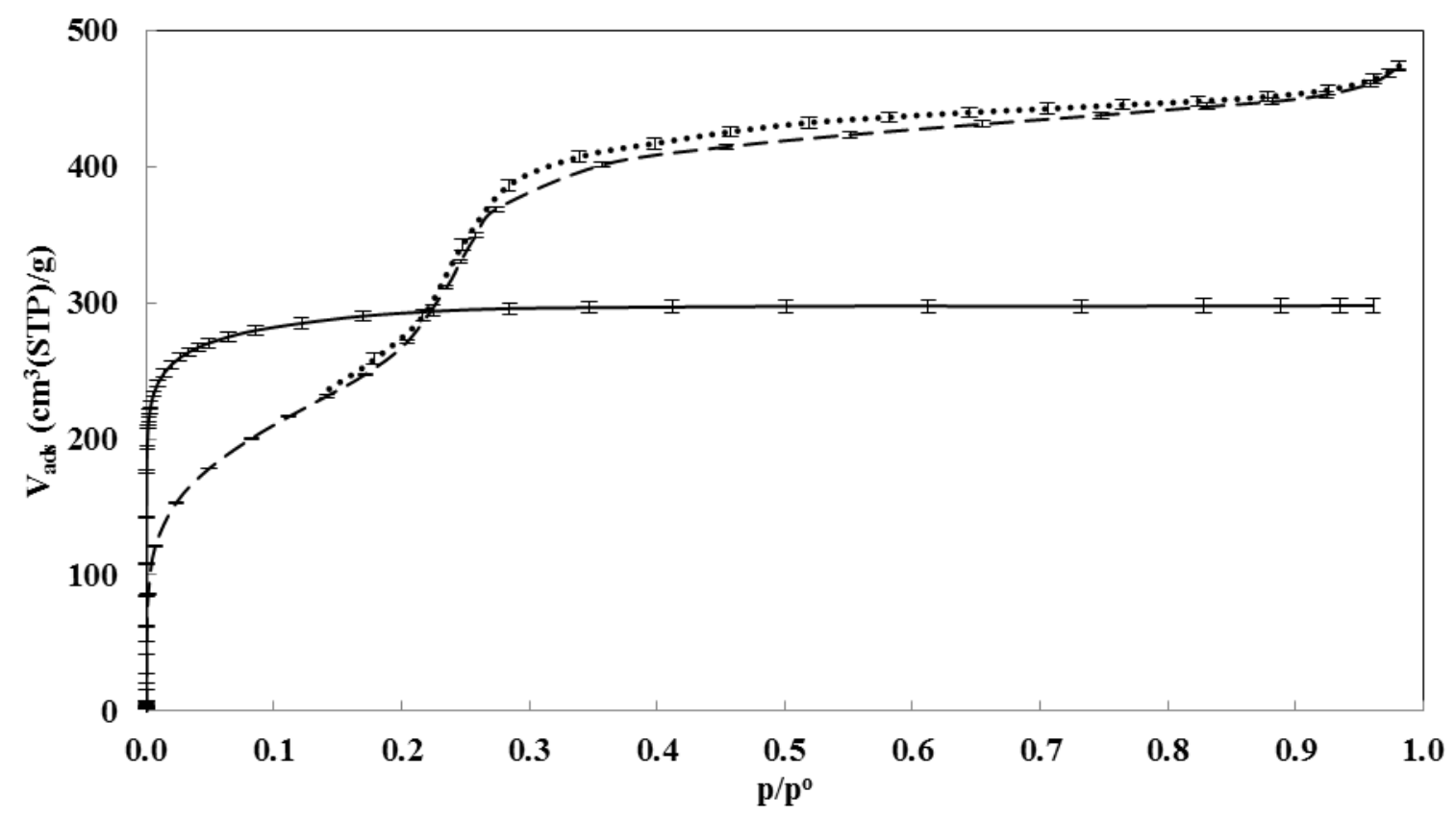

Figure 1. Nitrogen adsorption isotherm for microporous carbon cloth FM1/250 (-) and mesoporous MCM-41 (adsorption: - -, desorption: •••) showing uncertainty in amounts adsorbed for each equilibrium relative pressure. Each sample was degassed at $200{ }^{\circ} \mathrm{C}$ and 0.1 $\mathrm{mPa}$ for $8 \mathrm{~h}$ 


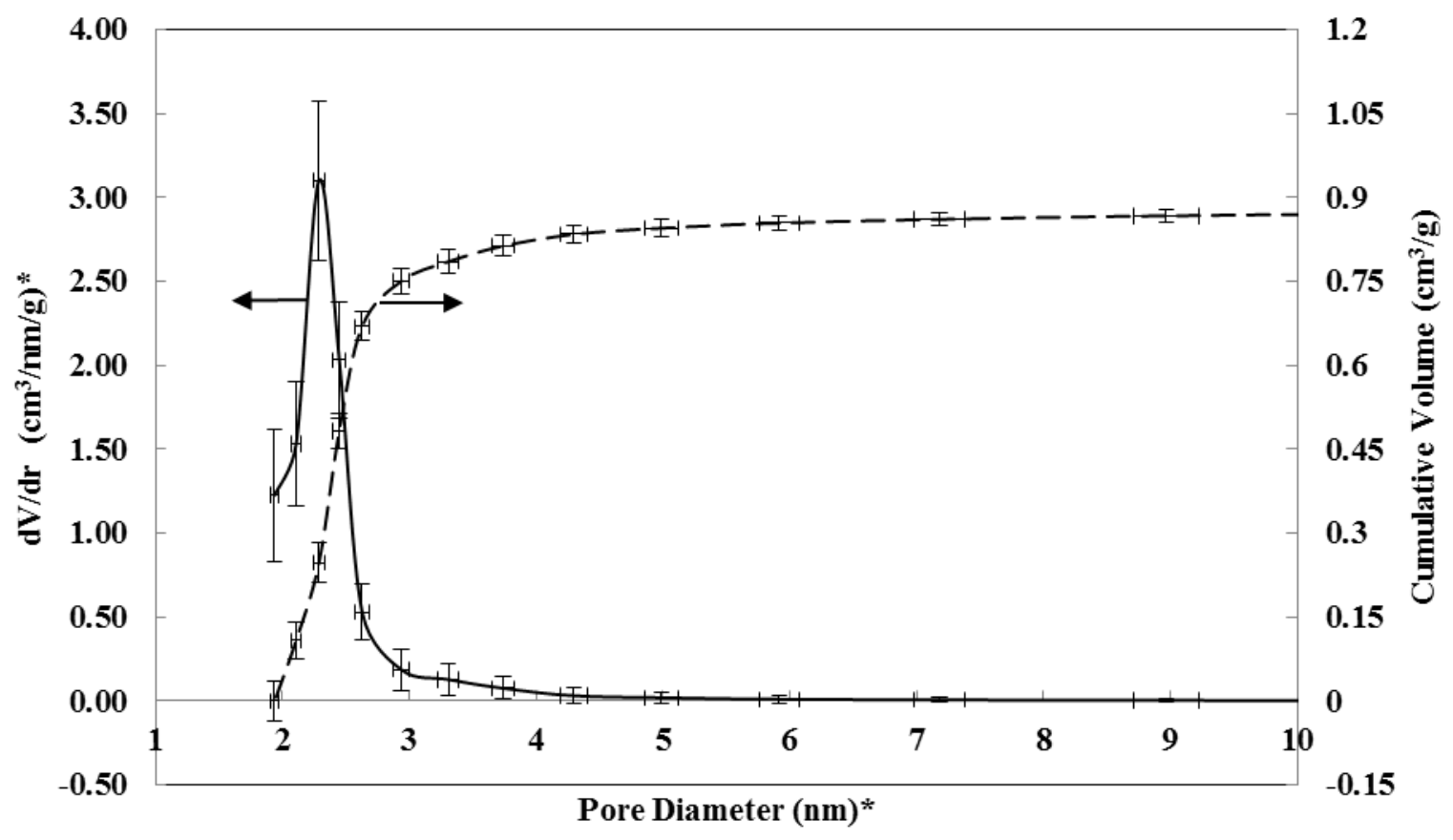

Figure 2. Calculated cumulative (liquid) volume adsorbed $(--)$, PSD $(-)$, and related uncertainties for mesoporous MCM-41

* Derivative is calculated based on pore radius but it is plotted against pore diameter to be consistent with next micropore section. 


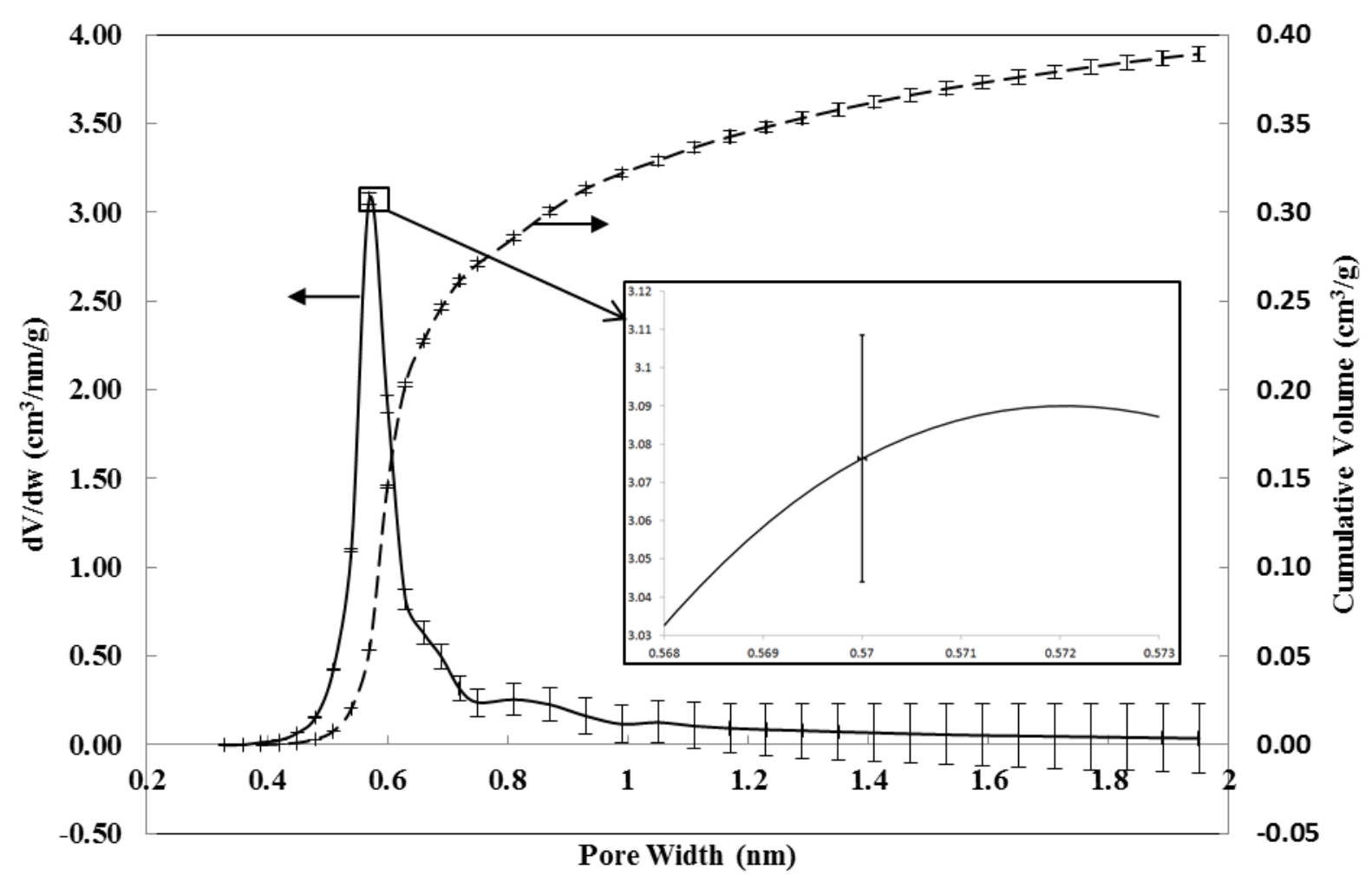

Figure 3. Calculated cumulative (liquid) volume adsorbed (- -), PSD (-), and related uncertainties for microporous carbon cloth FM1/250 

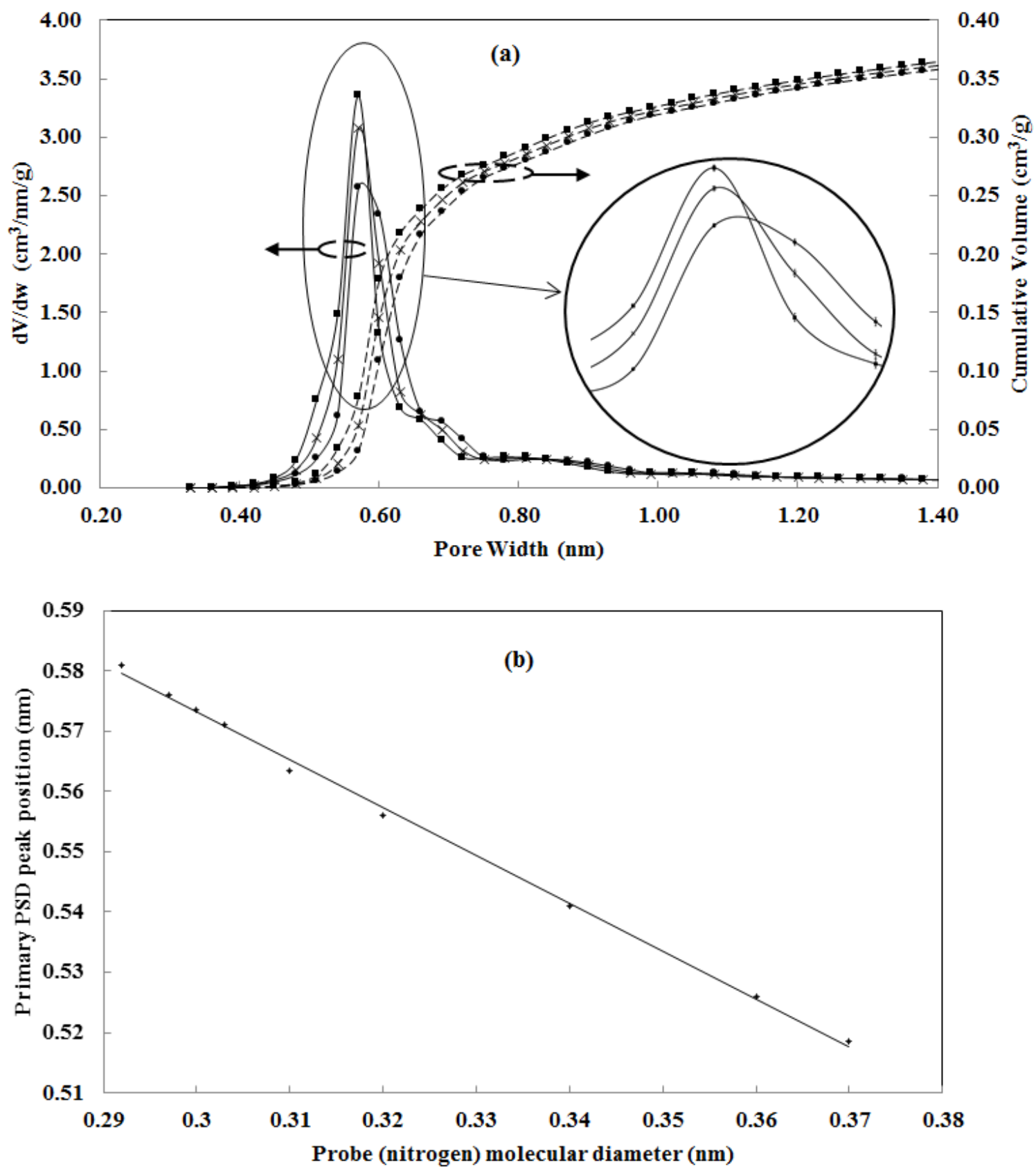

Figure 4. (a) Calculated cumulative volume (- -) and PSD (-) for microporous carbon cloth using different nitrogen molecular diameters: $0.297(\bullet), 0.3(\mathrm{x})$, and $0.303(\boldsymbol{\bullet})$. The inset shows the subtle increase in (apparent) mean pore diameter for the smallest probe dimension, $0.297 \mathrm{~nm}$; (b) effect of probe molecular diameter on the position of the main peak 